\title{
ESTIMATES ON THE GAP IN BULLEN'S INEQUALITY
}

\section{Flavia-Corina Mitroi-Symeonidis, Nicuşor Minculete and Marek NIEZGODA}

Abstract. Some companions and estimates of the gap in Bullen's inequality are shown. The results are applied to special means and connections with the methods of computational fluid dynamics are indicated.

Mathematics subject classification (2010): 26A51, 26D15. function.

Keywords and phrases: Bullen's inequality, Grüss inequality, Hermite-Hadamard inequality, convex

\section{REFERENCES}

[1] A. M. Acu AND H. Gonska, On Bullen's and related inequalities, General Mathematics, 22, 1 (2014), 19-26.

[2] M. KlaričIĆ BAKula, J. PeČArić And J. Perić, Extensions of the Hermite-Hadamard inequality with applications, Math. Inequal. Appl., 15, 4 (2012), 899-921.

[3] P. S. Bullen, Error estimates for some elementary quadrature rules, Univ. Beograd. Publ. Elektrotehn. Fak. Ser. Mat. Fiz., 602-633 (1978), 97-103.

[4] S. S. Dragomir, Bounds for the Normalized Jensen Functional, Bull. Austral. Math. Soc., 74, 3 (2006), 471-478.

[5] S. S. Dragomir, P. Cerone And A. Sofo, Some remarks on the midpoint rule in numerical integration, Studia Univ. Babes-Bolyai, Math., XLV, 1 (2000), 63-74.

[6] S. S. Dragomir, P. Cerone And A. Sofo, Some remarks on the trapezoid rule in numerical integration, Indian J. Pure Appl. Math., 31, 5 (2000), 475-494.

[7] S. S. Dragomir, Some Grüss type inequalities in inner product spaces, J. Inequal. Pure Appl. Math., 4, 2 (2003), Article 42.

[8] S. S. Dragomir And Ch. E. M. Pearce, Selected Topics on Hermite-Hadamard Inequalities and Applications, RGMIA Monographs, Victoria University, 2000.

[9] A. El FARISSI, Simple proof and refinement of Hermite-Hadamard inequality, J. Math. Inequal., 4, 3 (2010), 365-369.

[10] A. M. FINK, Bounds of the deviation of a function from its averages, Czechoslovak Math. J., 42, 117 (1992), 289-310.

[11] G. GRÜSS, Über das Maximum des absoluten Betrages von $\frac{1}{b-a} \int_{a}^{b} f(x) g(x) \mathrm{d} x$ $\frac{1}{(b-a)^{2}} \int_{a}^{b} f(x) \mathrm{d} x \int_{a}^{b} g(x) \mathrm{d} x$, Math. Z., 39, 1 (1935), 215-226.

[12] J. HADAMARD, Étude sur les propriétés des fonctions entières et en particulier d'une fonction considerée par Riemann, J. Math. Pures Appl. 58 (1893), 171-215.

[13] P. C. HammeR, The midpoint method of numerical integration, Math. Mag., 31 (1957/1958), 193 195.

[14] Ch. Hermite, Sur deux limites d'une intégrale définie, Mathesis, 3 (1883), 82.

[15] K. S. K. IYEngar, Note on an inequality, Math. Student, 6 (1938), 75-76.

[16] A. LuPAŞ, A generalization of Hadamard inequalities for convex functions, Univ. Beograd. Publ. Elektrotehn. Fak. Ser. Mat. Fiz. 544-576 (1976/1977), 115-121.

[17] G. S. MahaJani, A note on an inequality, Math. Student, 6 (1938), 125-128. 
[18] K. McGrattan, S. Hostikka, R. McDermott, J. Floyd , C. Weinschenk, K. Overholt, Fire Dynamics Simulator, Technical Reference Guide, Volume 1: Mathematical Model, NIST Special Publication 1018-1, 6th Ed., National Institute of Standards and Technology, Gaithersburg, MA, November 2013.

[19] N. Minculete, P. Dicu AND A. Ratiu, Two reverse inequalities of Bullen's inequalities, General Mathematics, 22, 1 (2014), 69-73.

[20] D. S. Mitrinović, J. PeČArić, A. M. Fink, Classical and new inequalities in analysis, Mathematics and its Applications (East European Series) 61, Kluwer Academic Publishers Group, Dordrecht, 1993.

[21] C. P. Niculescu And L.-E. Persson, Convex functions and their applications. A contemporary approach, Springer, 2006.

[22] M. NiEZGODA, A new inequality of Ostrowski-Grüss type and applications to some numerical quadrature rules, Comput. Math. Appl., 58 (2009), 589-596.

[23] M. NiezGodA, Translation-invariant maps and applications, J. Math. Anal. Appl., 354 (2009), 111124.

[24] J. PeČarić, F. Proschan And Y. L. Tong, Convex functions, partial orderings and statistical applications, Academic Press, New York, 1992.

[25] J. ŚANDor, Some integral inequalities, El. Math., 43 (1988), 177-180.

[26] P. M. VASIĆ AND I. B. LACKOviĆ, Some complements to the paper: On an inequality for convex functions, Univ. Beograd. Publ. Elektrotech. Fak. Ser. Mat. Fiz. 461-497 (1974), 63-66; Univ. Beograd. Publ. Elektrotehn. Fak. Ser. Mat. Fiz. 544-576 (1976), 59-62. 